\title{
Decreased level of consciousness in a toddler with overgrowth syndrome
}

\author{
Stephanie Fong, Alistair Scott, Dora Laczko \\ Faculty Reviewer: Dr Tamara Van Hooren, MD, FRCPC (Department of Paediatrics)
}

\section{ABSTRACT}

Physicians caring for patients with rare diseases face unique challenges in managing symptoms, ordering diagnostic tests, and providing patients and families with anticipatory guidance. We describe the case of a toddler with overgrowth syndrome, and previously known ophthalmological and neurological findings, presenting with decreased level of consciousness (LOC) following a fall. We highlight the extensive workup undertaken in a patient with symptoms spanning multiple systems but lacking a unifying diagnosis. In this case, rapid whole exome sequencing identified a de novo CACNA1A gene mutation encoding a calcium channel subunit, located within chromosome region 19p13.13. We explore 19p13.13 Microdeletion Syndrome and compare our patient's presentation to the cases described in the literature. Although our patient had several symptoms consistent with 19p13.13 Microdeletion Syndrome, others remain unexplained. This highlights the difficulty in determining a definitive diagnosis or treatment plan in the realm of rare diseases and emphasizes the need for further research into the disease process and development of novel therapies.

\section{PATIENT PRESENTATION}

A 2 years 10 months old male presents to hospital with decreased LOC following an unwitnessed fall. He was found facedown by a caregiver who described unusual movements but no clear seizure activity. Emergency medical services brought him to a local community hospital, during which he required support by bag mask ventilation.

The patient's past medical history was significant for global developmental delay, chronic overgrowth of undetermined etiology (weight average for 9 year old male, and height average for 5 year old male), retinal cone dysfunction, peripherally hypopigmented fundus, hypotonia, and autistic features. Specialists involved in his care included paediatric neurology, ophthalmology, endocrinology and medical genetics at multiple academic medical institutions, yet he lacked a unifying diagnosis. Prior to the unwitnessed fall the patient had had a 3-day history of cough but had otherwise been well.

Upon presentation to the local community emergency department, the patient was tachycardic and required supplemental oxygen. He required intubation for decreased respiratory effort, and was transferred to a paediatric critical care unit (PCCU) at the nearest tertiary care centre, where he remained intubated for 10 days. After successful extubation he was transferred to a general paediatric inpatient unit for further work-up and management.

\section{INVESTIGATIONS AND MANAGEMENT}

On initial presentation to the PCCU, the patient was started on empiric broad-spectrum antibiotics and antiviral treatment for possible meningitis or encephalitis. Infectious workup, including cultures of urine, blood, and cerebral spinal fluid (CSF), were negative. $\mathrm{CSF}$ polymerase chain reaction (PCR) was negative for enterovirus and herpes.

A computed tomography (CT) of the head ruled out any acute intracranial abnormality. Magnetic resonance imaging (MRI) of the head identified restricted diffusion involving parietal and occipital cortices bilaterally in a symmetrical fashion. Repeat MRI head 4 weeks later was normal. Ophthalmologic examination was unchanged from previous.

Electroencephalogram (EEG) findings were nonspecific but suggested moderate to diffuse encephalopathy with left occipital spike and wave discharges associated with increased risk of focal seizures. Paediatric neurology started the patient on phenytoin and levetiracetam following seizure in the PCCU. During hospitalization, he developed moderate choreiform movements of all four limbs and oculogyric phenomena involving the upward rolling of the eyes. Phenytoin was discontinued and valproic acid started. Levetiracetam was weaned in discussion with his family due to concern that the anti-epileptic was contributing to the patient's chorea.

Although the patient was feeding orally prior to his acute change in LOC, in hospital he developed feeding intolerance on nasogastric (NG) tube feeds and ultimately required gastrostomy tube insertion.

Previous genetic testing included normal chromosomal microarray, Fragile X testing, Simpson-Golabi-Behmel testing, isoelectric focusing, very long chain fatty acids, Bardet-Biedl and Alström panels, and GeneDx overgrowth panel. While in hospital, the patient was followed by the medical genetics team who recommended several additional investigations. Acylcarnitine profile and urine organic acids were normal. Skeletal survey was unremarkable with no dysplasia, metabolic bone disease, congenital malformation, or trauma identified. Muscle biopsy was performed for mitochondrial studies with results pending. Rapid whole exome sequencing identified a de novo pathogenic variant in CACNA1A, the gene responsible for calcium voltage-gated channel subunit alpha 1A.

\section{DIAGNOSIS AND FOLLOW-UP}

Despite the identification of a de novo CACNAlA gene mutation, there is an association between overgrowth syndrome and mutations in this gene region. CACNA1A loss-of-function mutations have been associated with symptoms including cognitive impair- 
ment, epileptic encephalopathy, nystagmus, autism, and ataxia. ${ }^{1-7}$

The patient was discharged with a diagnosis of encephalopathy, and started on anticonvulsive therapy. Both seizures and choreiform movement disorder were felt to most likely represent progression of his underlying diagnosis. With diminished developmental abilities and inability to orally feed, it was considered that his encephalopathy may be progressive. Community supports at discharge included occupational therapy, physical therapy, speech language pathology, and enteral feeding. Paediatric neurology, medical genetics, and community paediatrics were to continue as part of his treatment team with scheduled follow-up appointments.

In follow-up with the patient's family post-discharge, antiepileptic therapy had been weaned, and shortly following his choreiform movement disorder resolved. Though not completely back to his pre-admission baseline developmentally, his family had noted some improvements including the ability to safely tolerate some oral feed.

\section{DISCUSSION}

In 2009, a case report described a child who presented with syndromic craniosynostosis and microdeletion of chromosomal region 19 p13.13. ${ }^{8}$ Another case was published in the same year of a child with psychomotor delay, overgrowth, and infantile spasms, in which the CACNA1A gene, located within region 19p13.13, was mutated. ${ }^{1}$ CACNA1A encodes the main alpha 1A subunit of a voltage-dependent calcium channel, and mutations in this gene have been associated with epilepsy and implicated in episodic ataxia type 2 , spinocerebellar ataxia type 6 , and familial hemiplegic migraine. $^{2-7}$

With the description of another 5 cases of 19p13.13 microdeletion, each with similar presentations, including overgrowth, macrocephaly, intellectual delay and gastrointestinal problems, a unique syndrome was proposed, termed 19p13.13 Microdeletion Syndrome. ${ }^{1,8-11}$ Additional cases have been described of children with microdeletions spanning the 19p13.12 to 19p13.2 region (which includes the CACNA1A gene) who presented with syndromic craniosynostosis, epilepsy, and mental retardation. ${ }^{12}$

Microdeletions of neighboring region 19p13.12 have been associated with similar phenotypes such as Malan syndrome (Sotos syndrome 2), which present with features such as developmental delay, macrocephaly, central nervous abnormalities, overgrowth, and cephalic malformations. ${ }^{13,14}$ Deletions in the 19 p13 region are exceedingly rare; as such, only five case reports have been described., ${ }^{1,-11}$ But they, and this case, present a unique opportunity for research into the roles of genes such as CACNA1A in normal physical and intellectual development.

This case report describes a 2 years 10 months old male with a unique constellation of symptoms including developmental delay, overgrowth with undetermined etiology, retinal cone dysfunction, peripherally hypopigmented fundus, hypotonia, and autistic features. On admission to hospital he was found to have seizure activity on EEG and developed choreiform movements of all four limbs and oculogyric phenomena. Through rapid whole exome sequencing, a variant in CACNA1A, the gene encoding calcium voltage-gated channel subunit alpha-1A, was identified.
A limited number of case reports describing CACNA1A loss-offunction mutations have found associations between these mutations and episodic ataxia type 2 , spinocerebellar ataxia type 6 , and familial hemiplegic migraine..$^{2-7}$ Some of this patient's symptoms are consistent with previous reports, including developmental delay, hypotonia, and overgrowth syndrome. ${ }^{2}$ In addition, the development of seizure activity is supported by literature as CACNA1A mutations have been associated with epilepsy and febrile seizures. ${ }^{1,2}$ However the patient had other symptoms that remain unexplained. The clear retinal findings in this patient have not been previously reported in the literature. Additionally, his movement disorder is not a previously described finding, though it's resolution post-discharge may imply it is not associated with a CACNAlA mutation.

As limited literature exists on CACNA1A loss-of-function mutations, this genetic finding brought little clarity to the underlying disease process causing the patient's symptoms. In addition, there is still much that remains unknown, including the expected longterm progression of this patient's disease.

This case report highlights the complexities parents and families of children with rare diseases often face as they search for a diagnosis. Children with rare diseases often require frequent medical visits with specialists and are subjected to many diagnostic investigations, as presented in this report. This can be both emotionally and financially stressful for families as they care for their loved one without receiving a clear definitive diagnosis or treatment plan. This is made more stressful by the fact that rare diseases are associated with high morbidity and mortality. ${ }^{15,16}$

\section{CONCLUSION}

Clinicians should be aware of the significant impact that undiagnosed medical conditions have on the lives of paediatric patients and those who care for them. Clear communication between caregivers and the healthcare team, as well as between specialists, is an essential component of care for patients with complex medical conditions where management approaches may be limited or unknown. Further study into 19p13.13 Microdeletion Syndrome through basic science research into its mechanisms of pathogenesis and publication of observation and investigation of affected individuals is recommended to gain a better understanding of the role of CACNA1A in human development and lead to the development of novel therapeutics for this disorder.

\section{REFERENCES}

1. Auvin S, Holder-Espinasse M, Lamblin MD, Andrieux J. Array-CGH detection of a de novo 0.7-Mb deletion in 19p13.13 including CACNA1A associated with mental retardation and epilepsy with infantile spasms. Epilepsia. 2009; 50(11): p. 25015.

2. Jouvenceau A, Eunson LH, Spauschus A, Ramesh V, Zuberi SM, Kullmann DM, et al. Human epilepsy associated with dysfunction of the brain P/Q-type calcium channel. The Lancet. 2001 September; 358(9284): p. 801-7.

3. Ophoff RA, Terwindt GM, Vergouwe MN, van Eijk R, Oefner PJ, Hoffman SMG, et al. Familial Hemiplegic Migraine and Episodic Ataxia Type-2 Are Caused by Mutations in the Ca2 
Channel Gene CACNL1A4. Cell. 1996; 87(3): p. 543-52.

4. Beauvais K, Riant F, De Barace C, Alain F. New CACNA1A

Gene Mutation in a Case of Familial Hemiplegic Migraine with Status epilepticus. European Neurology. 2004 July; 52(1): p. 58-61.

5. Chioza B, Wilkie H, Nashef L, Blower J, McCormick D, Sham $\mathrm{P}$, et al. Association between the la calcium channel gene CACNA1A and idiopathic generalized epilepsy. Neurology. 2001 May; 56(9): p. 1245-6.

6. Kors EE, Vanmolkot KRJ, Kumlien E, Haan J, Raininko R, Flink R, et al. Childhood epilepsy, familial hemiplegic migraine, cerebellar ataxia, and a new CACNA1A mutation. Neurology. 2004 September; 63(6): p. 1136-7.

7. Guerin AA, Feigenbaum A, Donner EJ, Yoon G. Stepwise Developmental Regression Associated With Novel CACNA1A Mutation. Paediatric Neurology. 2008; 39(5): p. 363-4.

8. Lysy PA, Ravoet M, Wustefeld S, Bernard P, Nassogne MC, Wyns E, et al. A New Case of Syndromic Craniosynostosis With Cryptic 19p13.2-p13.13 Deletion. American Journal of Medical Genetics. 2009; 149(11): p. 2564-8.

9. Dolan M, Mendelsohn NJ, Pierpont ME, Schimmenti LA, Berry SA, Hirsch B. A novel microdeletion/microduplication syndrome of 19p13.13. Genetics in Medicine. 2010 August; 12(8): p. 503-11.

10. Nimmakayalu M, Horton VK, Darbro B, Patil SR, Alsayouf H, Keppler-Noreuil K, et al. Apparent Germline Mosaicism for a Novel 19p13.13 Deletion Disrupting NFIX and CACNA1A. American Journal of Medical Genetics. 2013; 161(5): p. 1105-9.

11. Jorge R, Silva C, Agueda S, Doria S, Leao M. Intellectual Disability and Overgrowth-A New Case of 19p13.13 Microdeletion Syndrome with Digital Abnormalities. American Journal of Medical Genetics. 2015 September; 167(11): p. 2839-43.

12. Lyon SM, Waggoner D, Halbach S, Thorland EC, Khorasani L, Reid RR. Syndromic craniosynostosis associated with microdeletion of chromosome 19p13.12-19p13.2. Genes \& Diseases. 2015; 2(4): p. 347-52.

13. Jezela-Stanek A, Kucharczyk M, Falana K, Jurkiewicz D, Mlynek M, Wicher D, et al. Malan syndrome (Sotos syndrome 2) in two patients with 19p13.2 deletion encompassing NFIX gene and novel NFIX sequence variant. Biomed Pap Med Fac Univ Palacky Olomouc Czech Repub. 2016 March; 160(1): p. 161-7.

14. Bonaglia MC, Marelli S, Novara F, Commodaro S, Borgatti R, Minardo G, et al. Genotype-phenotype relationship in three cases with overlapping 19p13.12 microdeletions. European Journal of Human Genetics. 2010 July; 18(12): p. 1302-9.

15. Canadian Organization for Rare Disorders. Canadian Organization for Rare Disorders. [Internet]. [cited 2017 July. Available from: www.raredisorders.ca/documents/ CORDFundraisingToolKit.pdf.

16. Spillmann RC, McConkie-Rosell A, Pena L, Yong-Hui J, Undiagnosed Diseases Network, Schoch K, et al. A window into living with an undiagnosed disease: illness narratives from the Undiagnosed Diseases Network. Orphanet Journal of Rare Diseases. 2017 April; 12(1): p. 71 